\title{
Comparison of the Net Work Output between Stirling and Ericsson Cycles
}

\author{
Rui F. Costa ${ }^{1}$ and Brendan D. MacDonald ${ }^{2, *}$ (D) \\ 1 Department of Mechanical Engineering, University of Coimbra, Rua Luís Reis Santos, \\ 3030-788 Coimbra, Portugal; ruicosta_94@hotmail.com \\ 2 Faculty of Engineering and Applied Science, University of Ontario Institute of Technology, \\ 2000 Simcoe Street North, Oshawa, ON L1H 7K4, Canada \\ * Correspondence: brendan.macdonald@uoit.ca; Tel.: +1-905-721-8668 (ext. 5716)
}

Received: 22 February 2018; Accepted: 14 March 2018; Published: 16 March 2018

\begin{abstract}
In this paper, we compare Stirling and Ericsson cycles to determine which engine produces greater net work output for various situations. Both cycles are for external heat engines that utilize regenerators, where the difference is the nature of the regeneration process, which is constant volume for Stirling and constant pressure for Ericsson. This difference alters the performance characteristics of the two engines drastically, and our comparison reveals which one produces greater net work output based on the thermodynamic parameters. The net work output equations are derived and analysed for three different scenarios: (i) equal mass and temperature limits; (ii) equal mass and pressure or volume; and (iii) equal temperature and pressure or volume limits. The comparison is performed by calculating when both cycles produce equal net work output and then analysing which one produces greater net work output based on how the parameters are varied. In general, the results demonstrate that Stirling engines produce more net work output at higher pressures and lower volumes, and Ericsson engines produce more net work output at lower pressures and higher volumes. For certain scenarios, threshold values are calculated to illustrate precisely when one cycle produces more net work output than the other. This paper can be used to inform the design of the engines and to determine when a Stirling or Ericsson engine should be selected for a particular application.
\end{abstract}

Keywords: Stirling cycle; Ericsson cycle; heat engines; external heat engines; thermodynamics

\section{Introduction}

Due to an increasing worldwide demand for clean energy, there is a corresponding need for alternative technologies to provide reliable and sustainable energy to meet this demand. External heat engines, such as Stirling and Ericsson engines, offer the ability to use many different heat sources to provide reliable and sustainable power, such as solar thermal, biomass and waste heat. Stirling and Ericsson cycles are ideal thermodynamic cycles for external heat engines with regeneration, and both are considered to have the Carnot efficiency as their theoretical efficiency, but they accomplish this using different thermodynamic cycles, with isochoric (constant volume) and isobaric (constant pressure) regeneration, respectively; thus, they exhibit different performance characteristics.

Throughout the years, Stirling and Ericsson engines have been individually studied and developed. Stirling engines have been tested and analysed for three main configurations: the alpha-type [1,2], beta-type [3-6] and gamma-type [7-10]. There have been thorough reviews of the Stirling engine by Kongtragool and Wongwises [11], Thombare and Verma [12] and Wang et al. [13]. The research of the Ericsson engine is not as extensive as the Stirling engine, but the Ericsson cycle was examined in a past study [14], as well as an open cycle Ericsson engine equipped with valves was analysed [15-17]. The above research has been instrumental in the development and improvement of these engines, 
but there has yet to be a comparison between Stirling and Ericsson engines to reveal which one preforms better and produces greater net work output for which situations.

Some of the past work has included analyses that apply to both Stirling and Ericsson engines, such as the work by Kaushik and Kumar [18], Tyagi et al. [19] and Bădescu [20]. Creyx et al. [21] optimized an engine operating with an open Joule or Ericsson cycle adapted for biomass upgrading and compared it to a Stirling engine used for the same specific application. Wojewoda and Kazimierski [22] investigated an externally-heated valve Joule engine and compared its efficiency and power output to a Stirling engine. Feidt and Costea [23] compared a number of combined heat and power systems, where they grouped Stirling and Ericsson engines together, and used an exergetic optimization approach to maximize the exergy. Hachem et al. [24] made a comparison based on an exergetic analysis of a gamma-type Stirling engine and an open Joule cycle engine. Eames et al. [25] compared the performance of externally-heated and recuperated Joule hot-air cycle engines to externally-heated closed Stirling cycle engines. To date, there has not been a direct comparison of the Stirling and Ericsson cycles, and as a result, we lack a robust understanding of when it is more advantageous to use one over the other.

In this paper, we compare the Stirling and Ericsson cycles and determine when one is more advantageous than the other. Since the purpose of a heat engine is to convert heat energy to mechanical work, we base the comparison on determining the situations where one cycle has a higher net work output than the other. The working fluid is assumed to be an ideal gas with $P V=m R T$; thus, there are four main parameters that determine the net work output of both cycles: the pressure $(P)$, the volume $(V)$, the total mass of the working fluid $(m)$ and the temperature $(T)$. We determine the influence of each parameter by calculating the work produced by each cycle over a range of values while a subset of the other parameters is fixed. By calculating the values at which the work is equal for both cycles, we are able to determine over which range of parameters each cycle will produce more or less work relative to the other cycle. With this analysis, we are able to understand when one cycle is more advantageous than the other, which can be used to inform the design of the engines and determine when one should be selected over the other for a particular application.

\section{Theoretical Model}

The theoretical Stirling and Ericsson cycles both have isothermal heat addition and heat rejection processes. The main differences between them are the regeneration processes with two isochoric (constant volume) regeneration processes for the Stirling cycle and two isobaric (constant pressure) regeneration processes for the Ericsson cycle.

The comparison of both cycles is made by analysing in which cases one cycle produces more net work than the other. The expressions for the net work are derived for each cycle by integrating the expression for the work:

$$
d W=P d V
$$

\subsection{Stirling Cycle}

The net work of the Stirling cycle is calculated with the following integration, using the reference volumes that are listed in the $P-V$ diagram in Figure 1:

$$
W_{S}=\int_{V_{1}}^{V_{2}} P d V+\int_{V_{3}}^{V_{4}} P d V
$$

From Figure 1, we can denote the maximum and minimum volumes for the processes as:

$$
\begin{aligned}
& V_{1}=V_{4}=V_{\max } \\
& V_{2}=V_{3}=V_{\text {min }}
\end{aligned}
$$


Substituting the volumes in Equation (2) and rewriting the pressure according to the ideal gas relation, the net work of the Stirling cycle can be written as:

$$
W_{S}=\int_{V_{\max }}^{V_{\min }} \frac{m R T_{L}}{V} d V+\int_{V_{\max }}^{V_{\min }} \frac{m R T_{H}}{V} d V
$$

After integrating, we have:

$$
W_{S}=m R T_{L}[\ln V]_{V_{\max }}^{V_{\min }}+m R T_{H}[\ln V]_{V_{\min }}^{V_{\max }}
$$

Rearranging yields:

$$
W_{S}=m R\left(T_{H}-T_{L}\right) \ln \left(\frac{V_{\max }}{V_{\min }}\right)
$$

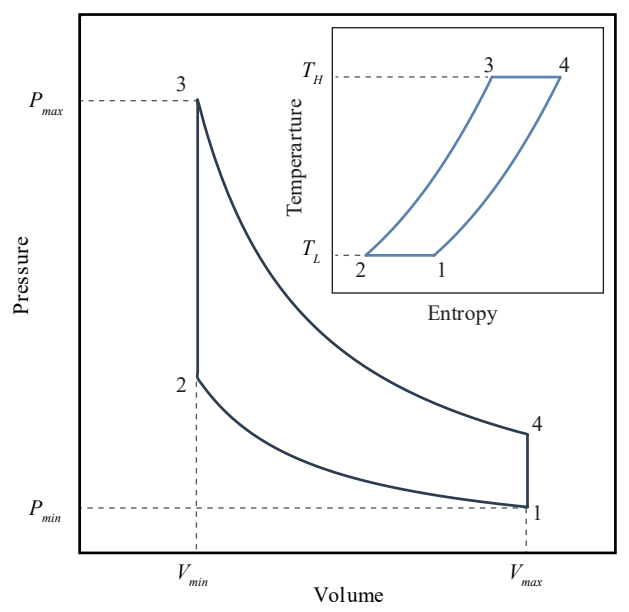

Figure 1. $P-V$ diagram of the Stirling cycle and $T$-s diagram (inset).

\subsection{Ericsson Cycle}

Similarly, integrating each process in the Ericsson cycle, with the volumes shown on the $P-V$ diagram in Figure 2, the net work is:

$$
W_{E}=\int_{V_{1}}^{V_{2}} P d V+\int_{V_{2}}^{V_{3}} P_{\max } d V+\int_{V_{3}}^{V_{4}} P d V+\int_{V_{4}}^{V_{1}} P_{\min } d V
$$

Based on the volumes shown in Figure 2 and the ideal gas relation, we can rewrite the volumes according to the maximum and minimum volumes and pressures as follows:

$$
\begin{aligned}
V_{1}=\frac{m R T_{L}}{P_{\min }} & =V_{\min }\left(\frac{P_{\max }}{P_{\min }}\right) \\
V_{2} & =V_{\min } \\
V_{3}=\frac{m R T_{H}}{P_{\max }} & =V_{\max }\left(\frac{P_{\min }}{P_{\max }}\right) \\
V_{4} & =V_{\max }
\end{aligned}
$$

Substituting these expressions into Equation (8) yields:

$$
W_{E}=\int_{V_{\min }\left(\frac{P_{\max }}{P_{\min }}\right)}^{V_{\min }} \frac{m R T_{L}}{V} d V+\int_{V_{\min }}^{V_{\max }\left(\frac{P_{\min }}{P_{\max }}\right)} P_{\max } d V+\int_{V_{\max }\left(\frac{P_{\min }}{P_{\max }}\right)}^{V_{\max }} \frac{m R T_{H}}{V} d V+\int_{V_{\max }}^{V_{\min }\left(\frac{P_{\max }}{P_{\min }}\right)} P_{\min } d V
$$


After integrating, we have:

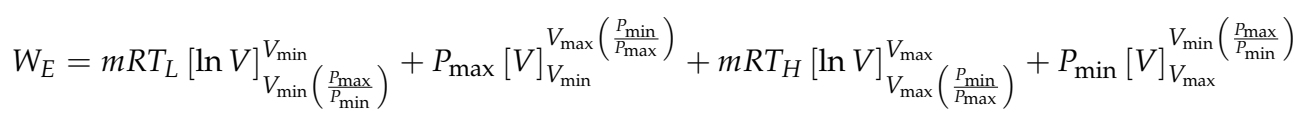

Rearranging yields:

$$
W_{E}=m R\left(T_{H}-T_{L}\right) \ln \left(\frac{P_{\max }}{P_{\min }}\right)
$$

Equations (7) and (15) give the net work for the Stirling and Ericsson cycles respectively and will be used as the basis for the comparison between the two cycles.

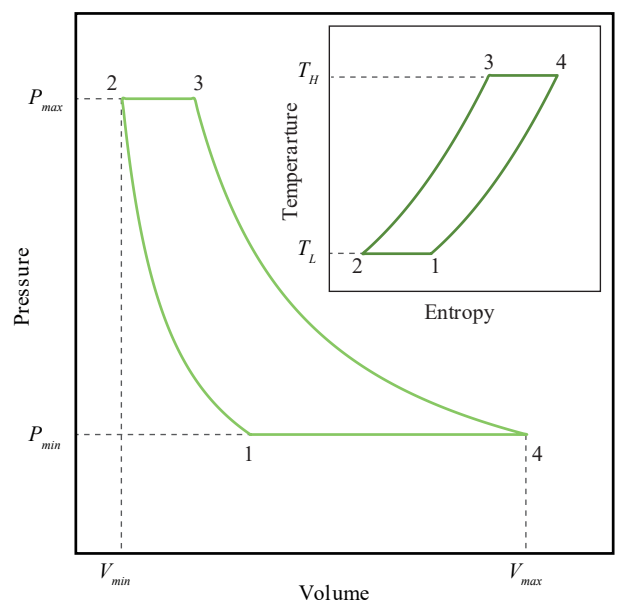

Figure 2. $P-V$ diagram of the Ericsson cycle and $T$-s diagram (inset).

\section{Results and Discussion}

Examination of Equations (7) and (15) shows that the difference in the net work between the Stirling and Ericsson cycles is the presence of the volume ratio in the Stirling work equation (Equation (7)) and pressure ratio in the Ericsson work equation (Equation (15)). This indicates that the net work output of the two cycles is equal when the volume ratio of the Stirling cycle is equal to the pressure ratio of the Ericsson. This fact alone does not reveal much about how the cycles compare with each other because the two cycles will often be compared when certain operating parameters are the same in each of the cycles. This leads us to analyse how the parameters influence the net work output of the cycles, to provide a more meaningful comparison.

The ideal gas relation limits how the thermodynamic parameters can vary throughout each of the cycles relative to one another. In order to understand how all of the parameters influence the net work output, both cycles are compared for three different scenarios: (i) equal mass and temperature limits, where pressure and volume ratios are varied; (ii) equal mass and pressure or volume ratios, where the temperature limits are varied; and (iii) equal temperature and pressure or volume limits, where the mass is varied. The three analyses provide a broad basis for comparison to assess which of the cycles are more favourable under which operating conditions.

\subsection{Comparison with Equivalent Mass and Temperature Limits}

For the first comparison, the two cycles are considered to have an equivalent mass of working fluid and the same high and low temperature limits. The fixed mass and temperature limits enable an analysis of how the pressure or volume ratio influences the net work output. The influence of the pressure ratio is analysed first, followed by the volume ratio. 
To analyse the influence of the pressure ratio on the net work output, the ideal gas relation is substituted into Equation (7):

$$
W_{S}=m R\left(T_{H}-T_{L}\right) \ln \left(\left(\frac{P_{\max }}{P_{\min }}\right)_{S} \frac{T_{L}}{T_{H}}\right)
$$

In order to calculate when the two cycles have equivalent net work output, Equations (15) and (16) can be equated and simplified by exploiting the equal mass and temperature limits, as follows:

$$
\left(\frac{P_{\max }}{P_{\min }}\right)_{S} \frac{T_{L}}{T_{H}}=\left(\frac{P_{\max }}{P_{\min }}\right)_{E}
$$

Since the temperature ratio in Equation (17) is always less than one, this result shows that in order to generate the equivalent net work output when mass and temperature limits are fixed, the Stirling cycle requires a higher pressure ratio than the Ericsson cycle. The pressure ratio of the Stirling cycle must be higher in proportion to the temperature ratio, as shown in Figure 3a. The solid lines in Figure 3a represent the equivalent net work output, while above the lines, the Ericsson cycle produces more work, and below the lines, the Stirling cycle produces more work. Figure 3a shows that for the same pressure ratio, the Ericsson cycle produces more net work output when the mass of the working fluid and the temperature limits are fixed.
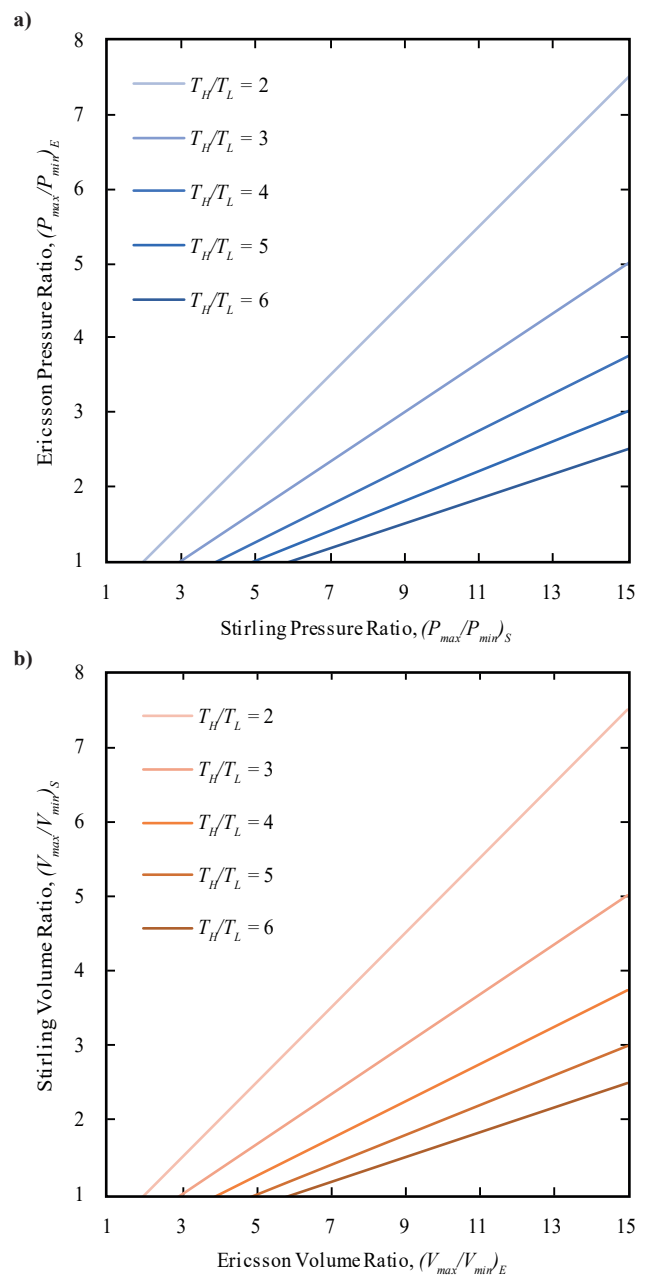

Figure 3. (a) Pressure ratio relationship and (b) volume ratio relationship between Stirling and Ericsson cycles, where the solid lines indicate equal net work output for equivalent mass and temperature limits. 
Similarly to the pressure ratio analysis, to analyse the influence of the volume ratio on the net work output, the ideal gas relation is substituted into Equation (15):

$$
W_{E}=m R\left(T_{H}-T_{L}\right) \ln \left(\left(\frac{V_{\max }}{V_{\min }}\right)_{E} \frac{T_{L}}{T_{H}}\right)
$$

In order to calculate when the two cycles have the equivalent net work output, Equations (7) and (18) can be equated and simplified by exploiting the equal mass and temperature limits, as follows:

$$
\left(\frac{V_{\max }}{V_{\min }}\right)_{S}=\left(\frac{V_{\max }}{V_{\min }}\right)_{E} \frac{T_{L}}{T_{H}}
$$

There is a reverse similarity in the results for the pressure and volume ratios. Since the temperature ratio in Equation (19) is always less than one, this result shows that in order to generate equivalent net work output when mass and temperature limits are fixed, the Ericsson cycle requires a higher volume ratio than the Stirling cycle. The volume ratio of the Stirling cycle must be higher in proportion to the temperature ratio, as shown in Figure $3 b$, which is the opposite of the pressure ratio results. Figure $3 \mathrm{~b}$ shows that for the same volume ratio, the Stirling cycle produces more net work output when the mass of the working fluid and the temperature limits are fixed.

The results of this analysis can be applied to a general rule about the selection of Stirling or Ericsson engines in practice, when the mass and temperature limits are fixed. If the pressure ratio is more limiting for the engine design than the engine size, an Ericsson engine is recommended. If the reverse is true and the engine size is a more critical constraint than the higher pressure ratio, a Stirling engine is recommended. To demonstrate the impact of this finding on the operating pressure and volume limits, two specific cases are examined in the following section.

\section{Fixed Pressure and Volume Limits}

In order to demonstrate how the results apply to the design of Stirling and Ericsson engines more directly, two specific cases are examined in this section: (i) one where the maximum pressure and volume are fixed; and (ii) one where the minimum pressure and volume are fixed. The specific operating parameters are listed in Table 1 , corresponding to a small stationary engine, to provide a representative example that can be scaled accordingly. The high temperature is set at $500{ }^{\circ} \mathrm{C}$ to enable a wide range of heat sources to provide the heat input, since many sustainable sources, such as solar thermal (concentrated), biomass and waste heat sources, can provide heat at this temperature.

For fixed maximum pressures and volumes, Equations (7) and (15) simplify to yield the same net work output for both cycles, as follows:

$$
W_{S}=W_{E}=m R\left(T_{H}-T_{L}\right) \ln \left(\frac{P_{\max } V_{\max }}{m R T_{H}}\right)
$$

The cycles can then be plotted on a $P-V$ diagram, as shown in Figure $4 \mathrm{a}$, to enable a comparison of the resulting minimum pressure and volume values. In both cases, Stirling's volume ratio is equal to Ericsson's pressure ratio to yield the same net work output. For this scenario, the Stirling cycle requires a lower minimum pressure value and higher minimum volume value, and the Ericsson cycle requires a lower minimum volume value and higher minimum pressure. This illustrates the need for the Stirling cycle to have a higher pressure ratio and the Ericsson cycle to have a higher volume ratio to generate the same amount of work.

Similarly, for fixed minimum pressures and volumes, Equations (7) and (15) simplify to yield the same net work output for both cycles, as follows:

$$
W_{S}=W_{E}=m R\left(T_{H}-T_{L}\right) \ln \left(\frac{m R T_{L}}{P_{\min } V_{\min }}\right)
$$


The resulting $P-V$ diagrams are plotted in Figure $4 \mathrm{~b}$, and as in the previous case, the Stirling volume ratio is equal to the Ericsson pressure ratio to yield the same net work output. For this scenario, the Stirling cycle requires a lower maximum volume value and higher maximum pressure, and the Ericsson cycle requires a higher maximum volume value and lower maximum pressure value. This confirms the need for the Stirling cycle to have a higher pressure ratio and the Ericsson cycle to have a higher volume ratio to generate the same amount of work. The $P-V$ diagram in Figure $4 \mathrm{~b}$ gives a visual demonstration of the requirement for the Stirling to have a higher pressure and the Ericsson to have a higher volume when the mass, temperature limits and minimum pressure and volume values are fixed.
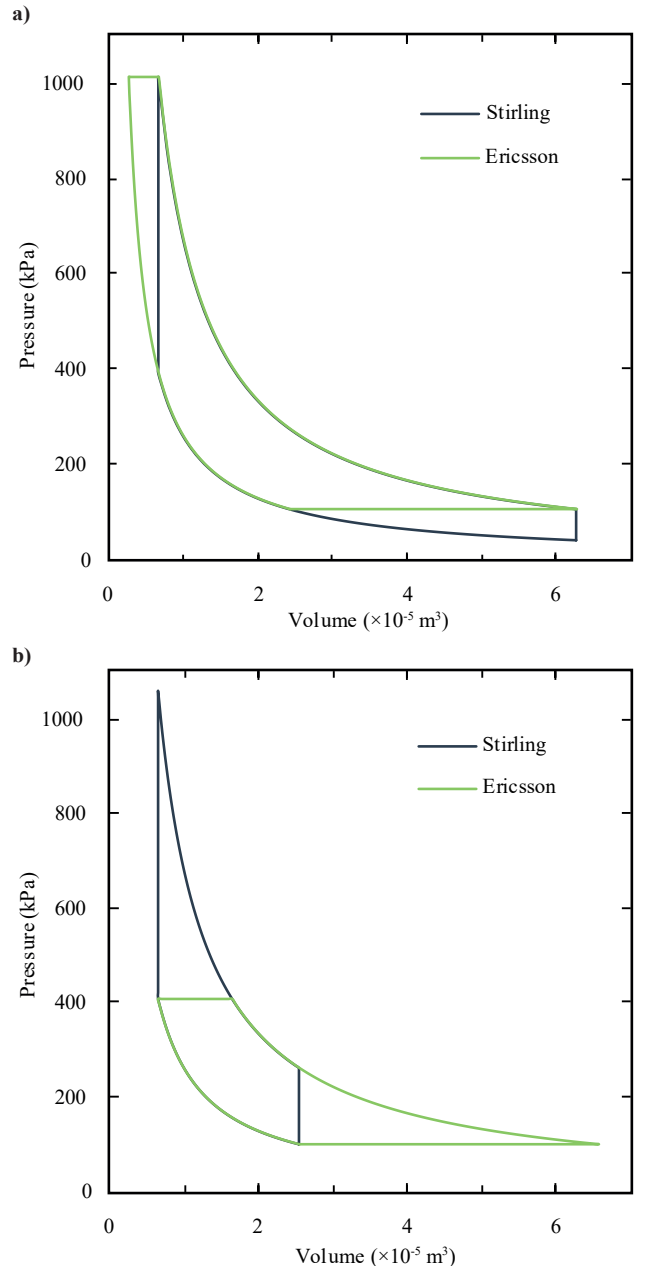

Figure 4. $P-V$ diagrams of Stirling and Ericsson cycles for fixed mass and temperature limits with (a) fixed maximum pressure and volume and (b) fixed minimum pressure and volume.

Table 1. Parameters for a small-scale engine (referring to Figure 4).

\begin{tabular}{ccc}
\hline Operating Parameters & Case (i) & Case (ii) \\
\hline Working fluid & air & air \\
$m(\mathrm{~kg})$ & 0.00003 & 0.00003 \\
$T_{H}\left({ }^{\circ} \mathrm{C}\right)$ & 500 & 500 \\
$T_{L}\left({ }^{\circ} \mathrm{C}\right)$ & 25 & 25 \\
$P_{\max }(\mathrm{kPa})$ & 1013.3 & - \\
$V_{\max }\left(\mathrm{m}^{3}\right)$ & $6.28 \times 10^{-5}$ & - \\
$P_{\min }(\mathrm{kPa})$ & - & 101.33 \\
$V_{\min }\left(\mathrm{m}^{3}\right)$ & - & $6.28 \times 10^{-6}$ \\
\hline
\end{tabular}




\subsection{Comparison with Equivalent Mass and Pressure or Volume Ratios}

For the second comparison, the two cycles are considered to have the same mass of the working fluid, and there are two cases: (i) one with the same pressure ratio; and (ii) one with the same volume ratio. Fixing the mass of the working fluid and the pressure or volume ratio allows for variation of the temperature limits. This case is useful for practical consideration because the low temperature limit is often governed by the atmospheric temperature and the high temperature is often governed by which heat source is selected.

\subsubsection{Equivalent Pressure Ratios}

In order to calculate when the two cycles have equivalent net work output for equal pressure ratio, Equations (15) and (16) can be equated and simplified by exploiting the equal mass of the working fluid and pressure ratio, as follows:

$$
\frac{P_{\max }}{P_{\min }}=\left(\frac{T_{L}}{T_{H}}\right)_{S}^{\frac{\left(T_{H}-T_{L}\right)_{S}}{\left(T_{H}-T_{L}\right)_{E}-\left(T_{H}-T_{L}\right)_{S}}}
$$

Unlike the previous case, in this case, the high and low temperatures appear individually, and not exclusively as a temperature ratio, so there is a need to fix the high or low temperatures in the analysis. Since the temperature of the heat sink is commonly more restrictive than the temperature of the heat source, the comparison is more relevant when the low temperature is fixed, and the engines are compared based on the temperature range of the heat sources (high temperature, $T_{H}$ ) that can be selected.

Equation (22) can be rearranged by fixing an equivalent low temperature $\left(T_{L, S}=T_{L, E}=T_{L}\right)$, as follows:

$$
T_{H, E}=T_{H, S}+\frac{\ln \left(\frac{T_{L}}{T_{H, S}}\right)}{\ln \left(\frac{P_{\max }}{P_{\min }}\right)}\left(T_{H, S}-T_{L}\right)
$$

This relation is plotted in Figure 5a for a low temperature value of $25^{\circ} \mathrm{C}$, with the solid lines showing the equivalent net work output, which illustrates that a Stirling cycle requires a higher high temperature value than the Ericsson to yield the same net work output when the mass and pressure ratio are equal. Below the solid line represents when the Stirling cycle produces more net work output, and correspondingly, above each solid line represents when the Ericsson cycle produces more net work output. The $P-V$ diagrams for this case are shown in Figure $5 \mathrm{~b}$ using the specific parameters listed in Table 2. It can be seen in Figure $5 b$ that for this scenario, the Stirling cycle requires a lower volume ratio to produce the same work as the Ericsson cycle, and as a result, the high temperature requirement for the Stirling cycle is higher. Conversely, the Ericsson cycle requires a higher volume ratio, which results in a lower high temperature requirement.

Table 2. Parameters for specific comparison (referring to Figures $5 b$ and $6 b$ ).

\begin{tabular}{ccc}
\hline Operating Parameters & Case (i) & Case (ii) \\
\hline Working fluid & air & air \\
$m(\mathrm{~kg})$ & 0.00003 & 0.00003 \\
$T_{L}\left({ }^{\circ} \mathrm{C}\right)$ & 25 & 25 \\
$T_{H, S}\left({ }^{\circ} \mathrm{C}\right)$ & 500 & - \\
$T_{H, E}\left({ }^{\circ} \mathrm{C}\right)$ & - & 500 \\
$P_{\max }(\mathrm{kPa})$ & 1013.3 & - \\
$P_{\min }(\mathrm{kPa})$ & 101.33 & - \\
$V_{\max }\left(\mathrm{m}^{3}\right)$ & - & $3.14 \times 10^{-5}$ \\
$V_{\min }\left(\mathrm{m}^{3}\right)$ & - & $3.14 \times 10^{-6}$ \\
\hline
\end{tabular}



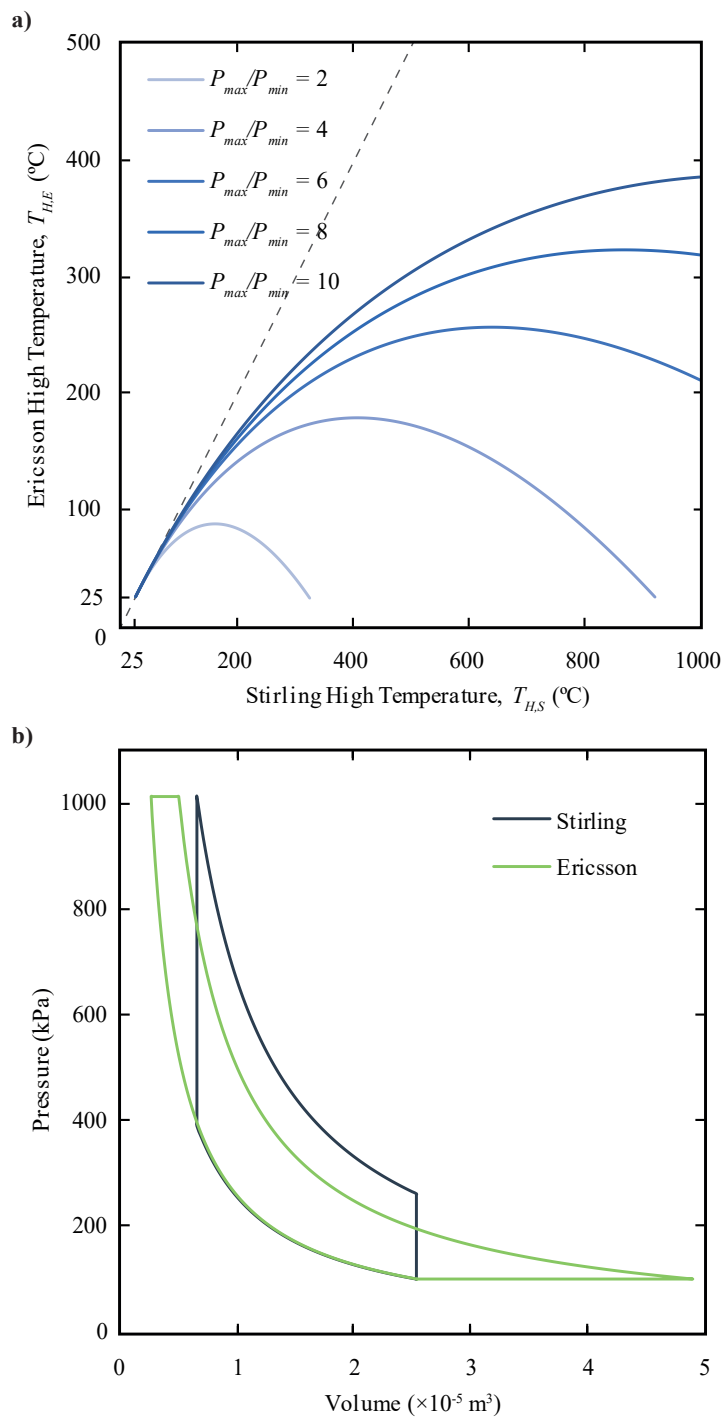

Figure 5. (a) High temperature relationship between Stirling and Ericsson cycles for fixed pressure ratios and $T_{L}=25^{\circ} \mathrm{C}$, with solid lines denoting equal net work output; and (b) $P$ - $V$ diagrams of Stirling and Ericsson cycles for fixed mass, pressure limits and low temperature for equal net work output.

\subsubsection{Equivalent Volume Ratios}

Similarly to the pressure ratio analysis, in order to calculate when the two cycles have equivalent net work output for equal volume ratio, Equations (7) and (18) can be equated and simplified by exploiting the equal mass of the working fluid and volume ratio, as follows:

$$
\frac{V_{\max }}{V_{\min }}=\left(\frac{T_{L}}{T_{H}}\right)_{E}^{\frac{\left(T_{H}-T_{L}\right)_{E}}{\left(T_{H}-T_{L}\right)_{S}-\left(T_{H}-T_{L}\right)_{E}}}
$$

Equation (24) can be rearranged by fixing an equivalent low temperature $\left(T_{L, S}=T_{L, E}=T_{L}\right)$, as follows:

$$
T_{H, S}=T_{H, E}+\frac{\ln \left(\frac{T_{L}}{T_{H, E}}\right)}{\ln \left(\frac{V_{\max }}{V_{\min }}\right)}\left(T_{H, E}-T_{L}\right)
$$


This relation is plotted in Figure 6 a for a low temperature value of $25^{\circ} \mathrm{C}$, with the solid lines showing the equivalent net work output, which illustrates that a Stirling cycle requires a lower high temperature value than the Ericsson to yield the same net work output when the mass and volume ratio are equal. Above the solid line represents when the Stirling cycle produces more net work output, and correspondingly, below each solid line represents when the Ericsson cycle produces more net work output. The $P-V$ diagrams for this case are shown in Figure $6 \mathrm{~b}$ using the specific parameters listed in Table 2. It can be seen in Figure $6 \mathrm{~b}$ that for this scenario, the Ericsson cycle requires a lower pressure ratio to produce the same work as the Stirling cycle, and as a result, the high temperature requirement for the Ericsson cycle is higher. Conversely, the Stirling cycle requires a higher pressure ratio, which results in a lower high temperature requirement.
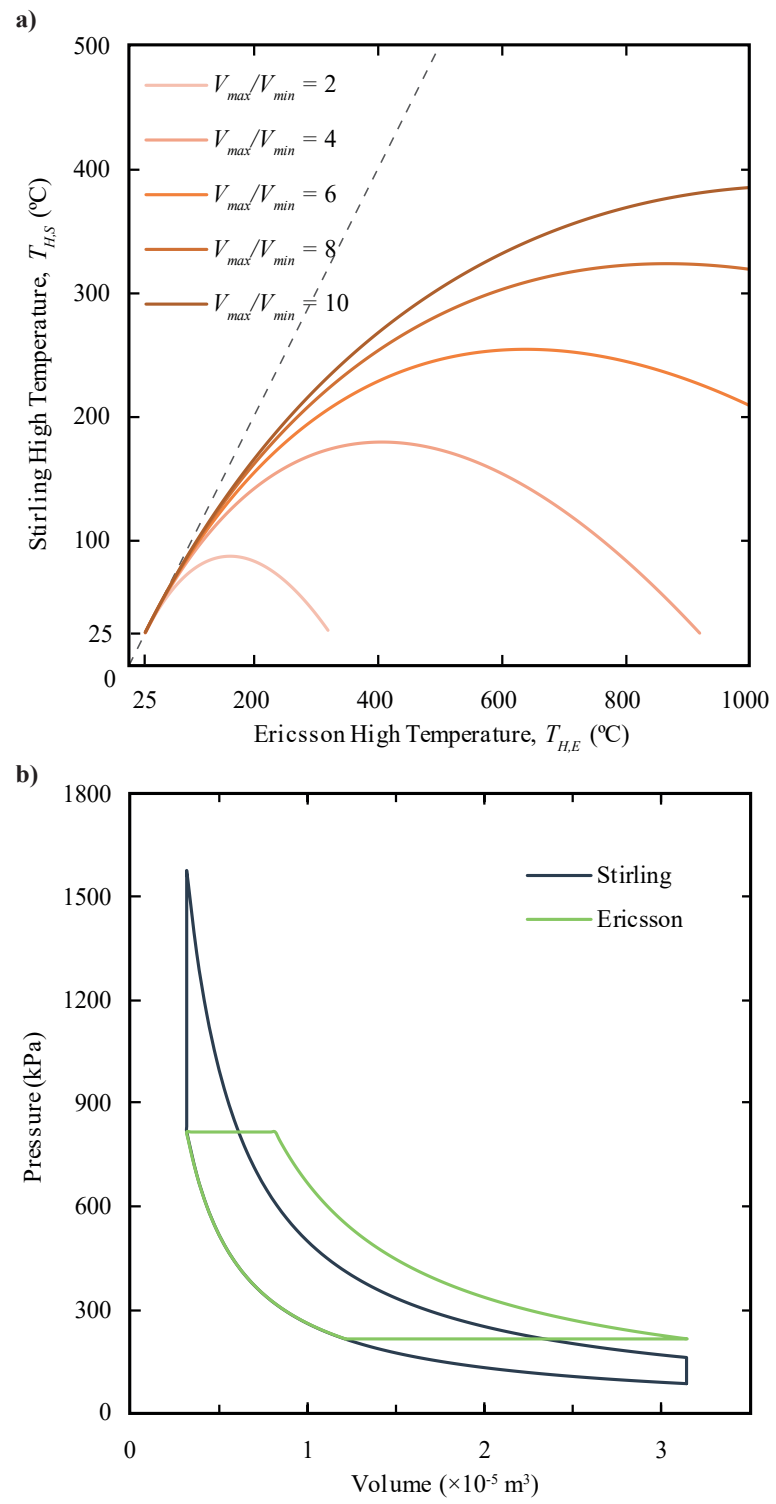

Figure 6. (a) High temperature relationship between Stirling and Ericsson cycles for fixed volume ratios and $T_{L}=25^{\circ} \mathrm{C}$, with solid lines denoting equal net work output; (b) $P$ - $V$ diagrams of Stirling and Ericsson cycles for fixed mass, volume limits and low temperature for equal net work output. 


\subsection{Comparison with Equivalent Temperature Limits and Pressure or Volume Ratios}

For the third comparison, the two cycles are considered to have the same high and low temperature limits, and there are two cases: (i) where the pressure ratio is equivalent and the maximum volume is fixed; and (ii) where the volume ratio is equivalent and the maximum pressure is fixed. Fixing the temperature limits along with either the pressure or volume ratios allows for variation of the mass of the working fluid. This situation is arguably more relevant for practical application since the mass of the working fluid is rarely a primary design consideration, and the other engine characteristics, such as operating pressures, size (volume) and the temperature of the available heat source and heat sink, are commonly more restrictive.

\subsubsection{Equivalent Pressure Ratio and Fixed Maximum Volume}

To compare the cycles with equivalent pressure ratios, Equations (15) and (16) can be simplified by exploiting the equal temperature limits and pressure ratio, and since the maximum volume is also fixed, the masses of the working fluid of the Stirling and Ericsson cycles are calculated using the maximum volume and minimum pressure, corresponding to Point 1 in Figure 1 and Point 4 in Figure 2, respectively, which yields:

$$
\begin{gathered}
W_{S}=V_{\max } P_{\min }\left(\frac{T_{H}}{T_{L}}-1\right) \ln \left(\frac{P_{\max } T_{L}}{P_{\min } T_{H}}\right) \\
W_{E}=V_{\max } P_{\min }\left(1-\frac{T_{L}}{T_{H}}\right) \ln \left(\frac{P_{\max }}{P_{\min }}\right)
\end{gathered}
$$

Non-dimensional net work can be achieved by dividing the net work by the maximum volume and minimum pressure, as follows:

$$
\begin{gathered}
\frac{W_{S}}{V_{\max } P_{\min }}=\left(\frac{T_{H}}{T_{L}}-1\right) \ln \left(\frac{P_{\max } T_{L}}{P_{\min } T_{H}}\right) \\
\frac{W_{E}}{V_{\max } P_{\min }}=\left(1-\frac{T_{L}}{T_{H}}\right) \ln \left(\frac{P_{\max }}{P_{\min }}\right)
\end{gathered}
$$

Equations (28) and (29) can be equalized to demonstrate the relationship between the temperature and pressure ratios when the cycles have equal net work output:

$$
\frac{P_{\max }}{P_{\min }}=\left(\frac{T_{L}}{T_{H}}\right)^{\frac{1}{T_{L}}-1}
$$

Equation (30) is plotted in Figure 7a with the solid line showing where the net work output of both cycles is equal. At the higher pressure ratios above the line, the Stirling cycle produces more net work, and at the lower pressure ratios below the line, the Ericsson cycle produces more net work. To show how this impacts a specific engine design, the inset shows the $P-V$ diagram corresponding to the parameters listed in Table 3 . The $P$ - $V$ diagram illustrates how the Ericsson cycle requires a lower minimum volume than the Stirling cycle to produce the same amount of net work output. This result ultimately indicates that for a given temperature ratio, with a size restriction (i.e., fixed maximum volume), there is a threshold pressure ratio that determines which cycle produces more net work. For example, as shown in Figure 7b, where Equations (28) and (29) are plotted, if the temperature ratio is fixed at 2.6, the threshold pressure ratio is 4.7, and below this value, an Ericsson cycle produces more net work output, while above it, a Stirling cycle produces more net work output. Correspondingly, as shown in the inset of Figure $7 \mathrm{~b}$, if the pressure ratio is fixed at 4.7 , the threshold temperature ratio is 2.6, and below this value, a Stirling cycle produces more net work output, while above it, an Ericsson cycle produces more net work output. Since engine design is commonly limited by a size restriction, 
the operating pressure limits and the temperature of the available heat source and sink, this result is a useful tool for determining which of the cycles should be used for specific scenarios.

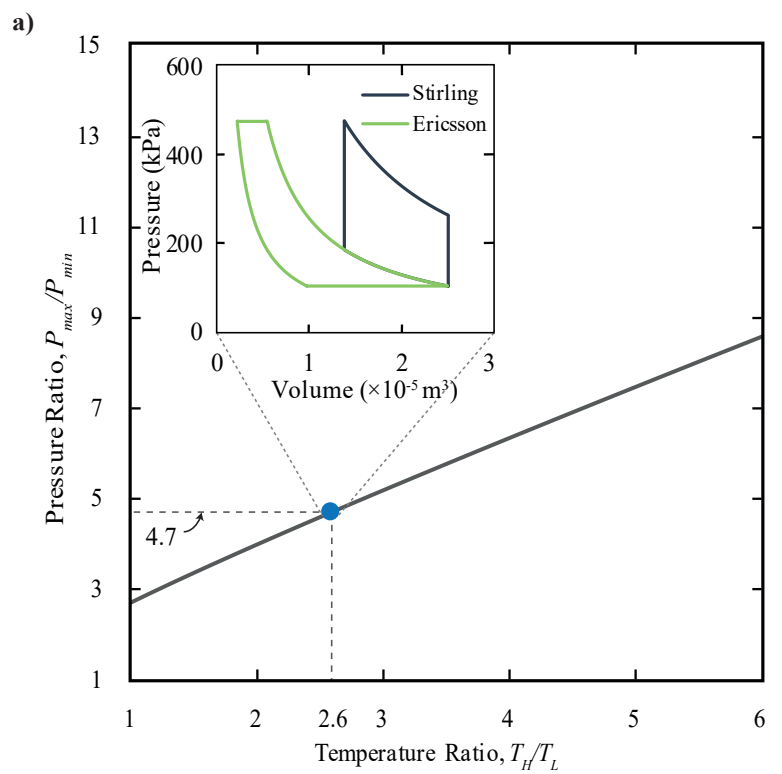

b)

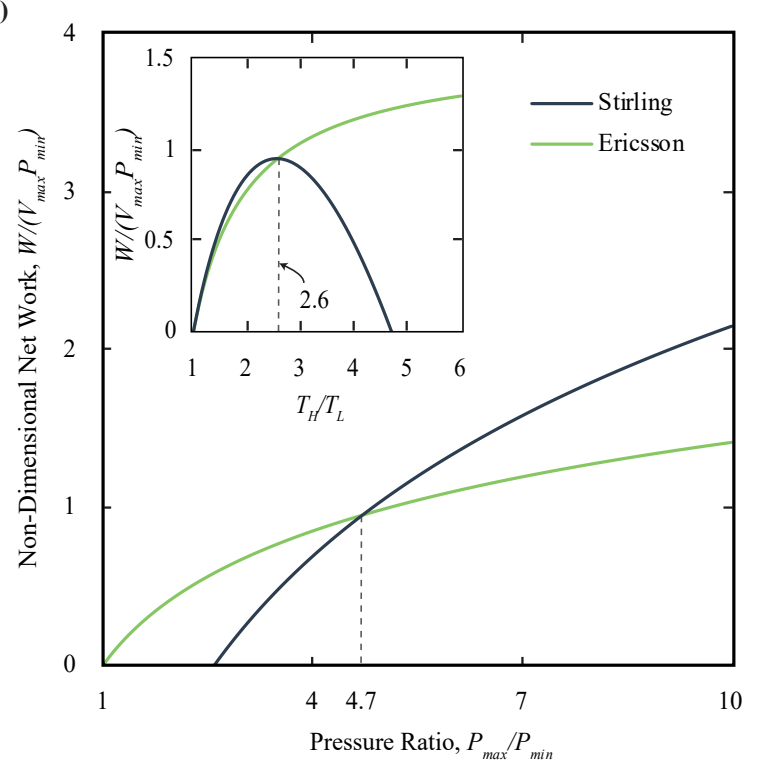

Figure 7. (a) Relationship between pressure and temperature ratio for equal net work output and $P-V$ diagram of Stirling and Ericsson cycles for fixed temperature and pressure limits and maximum volume for equal net work output (inset); (b) non-dimensional net work as a function of pressure ratio and temperature ratio (inset).

Table 3. Parameters for specific comparison (referring to Figures 7 and 8).

\begin{tabular}{ccc}
\hline Operating Parameters & Case (i) & Case (ii) \\
\hline Working fluid & air & air \\
$T_{H}\left({ }^{\circ} \mathrm{C}\right)$ & 500 & 500 \\
$T_{L}\left({ }^{\circ} \mathrm{C}\right)$ & 25 & 25 \\
$P_{\min }(\mathrm{kPa})$ & 101.33 & - \\
$V_{\max }\left(\mathrm{m}^{3}\right)$ & $2.51 \times 10^{-5}$ & - \\
$V_{\min }\left(\mathrm{m}^{3}\right)$ & - & $4.71 \times 10^{-6}$ \\
$P_{\max }(\mathrm{kPa})$ & - & 1013.3 \\
\hline
\end{tabular}




\subsubsection{Equivalent Volume Ratio and Fixed Maximum Pressure}

To compare the cycles with equivalent pressure ratios, Equations (7) and (18) can be simplified by exploiting the equal temperature limits and volume ratio, and since the maximum pressure is also fixed, the masses of the working fluid of the Stirling and Ericsson cycles are calculated using the maximum pressure and minimum volume, corresponding to Point 3 in Figure 1 and Point 2 in Figure 2, respectively, which yields:

$$
\begin{gathered}
W_{S}=P_{\max } V_{\min }\left(1-\frac{T_{L}}{T_{H}}\right) \ln \left(\frac{V_{\max }}{V_{\min }}\right) \\
W_{E}=P_{\max } V_{\min }\left(\frac{T_{H}}{T_{L}}-1\right) \ln \left(\frac{V_{\max } T_{L}}{V_{\min } T_{H}}\right)
\end{gathered}
$$

Non-dimensional net work can be achieved by dividing the net work by the maximum pressure and minimum volume, as follows:

$$
\begin{gathered}
\frac{W_{S}}{P_{\max } V_{\min }}=\left(1-\frac{T_{L}}{T_{H}}\right) \ln \left(\frac{V_{\max }}{V_{\min }}\right) \\
\frac{W_{E}}{P_{\max } V_{\min }}=\left(\frac{T_{H}}{T_{L}}-1\right) \ln \left(\frac{V_{\max } T_{L}}{V_{\min } T_{H}}\right)
\end{gathered}
$$

Equations (33) and (34) can be equalized to demonstrate the relationship between the temperature and volume ratios when the cycles have equal net work output:

$$
\frac{V_{\max }}{V_{\min }}=\left(\frac{T_{L}}{T_{H}}\right)^{\frac{1}{T_{L}}-1}
$$

Equation (35) is plotted in Figure 8a with the solid line showing where the net work output of both cycles is equal. The results here show similar patterns to the previous case with the maximum volume restriction. At the higher volume ratios above the line, the Ericsson cycle produces more net work, and at the lower volume ratios below the line, the Stirling cycle produces more net work. To show how this impacts a specific engine design, the inset shows the $P-V$ diagram corresponding to the parameters listed in Table 3. The $P-V$ diagram illustrates how the Stirling cycle requires a lower minimum pressure than the Ericsson cycle to produce the same amount of net work output. This result ultimately indicates that for a given temperature ratio, with a maximum pressure restriction, the volume ratio will determine which cycle produces more net work. For example, as shown in Figure 8b, where Equations (33) and (34) are plotted, if the temperature ratio is fixed at 2.6, the threshold volume ratio is 4.7 , and below this value, a Stirling cycle produces more net work output, while above it, an Ericsson cycle produces more net work output. Correspondingly, as shown in the inset of Figure $8 \mathrm{~b}$, if the volume ratio is fixed at 4.7 , the threshold temperature ratio is 2.6 , and below this value, an Ericsson cycle produces more net work output, while above it, a Stirling cycle produces more net work output. Similar to the previous case, since engine design is commonly limited by the attainable minimum and maximum volumes, the maximum operating pressure, and the temperature of the available heat source and sink, this result is a useful tool for determining which of the two cycles should be used for specific scenarios. This case is more applicable when the minimum volume is more critical, and the previous case is more applicable when the minimum pressure is more critical. 
a)

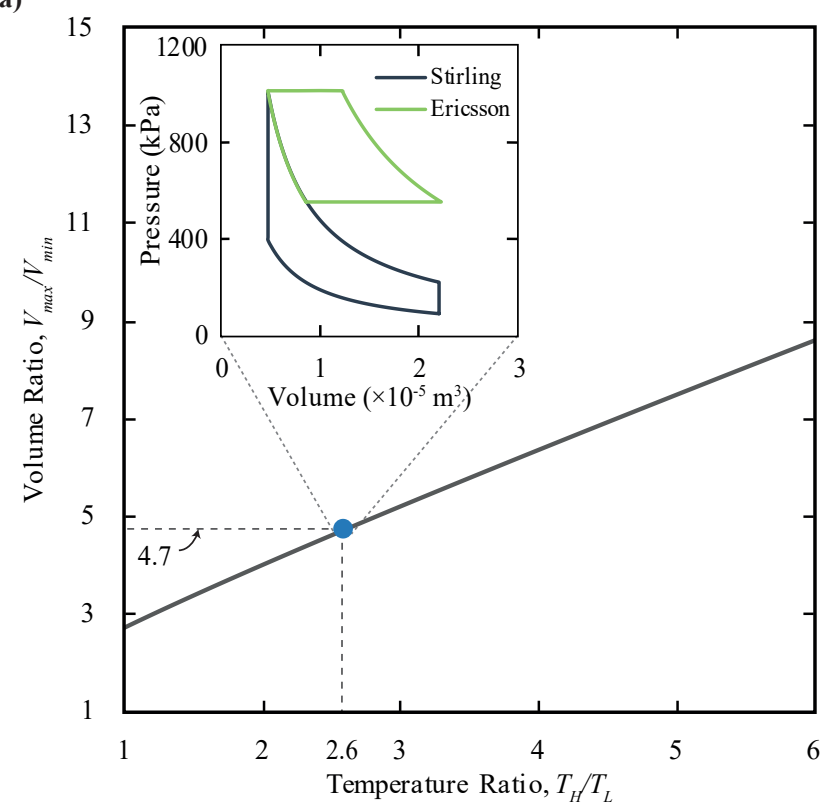

b)

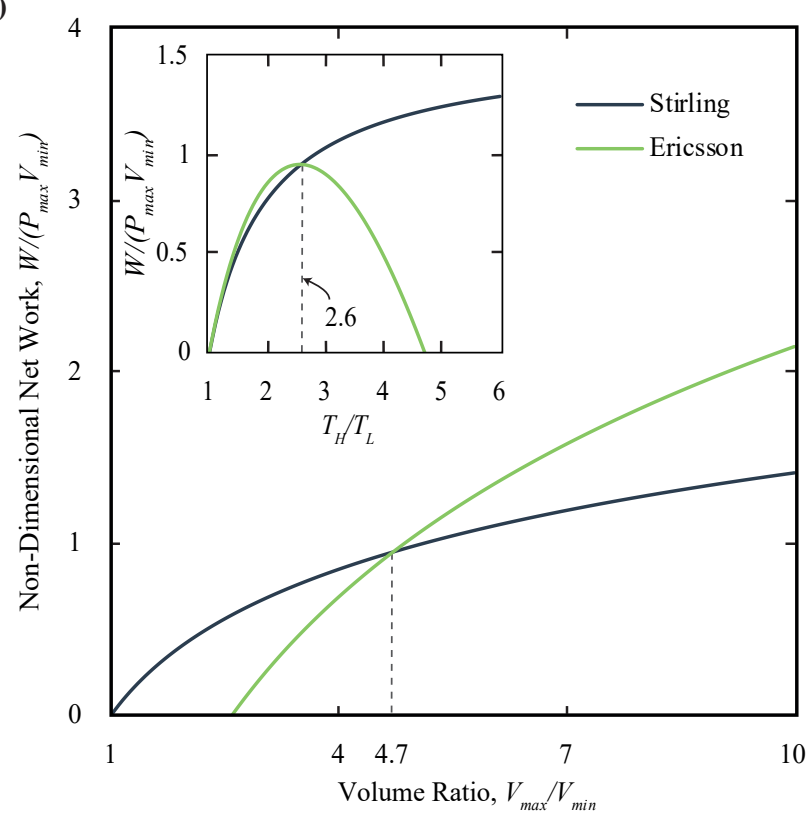

Figure 8. (a) Relationship between volume and temperature ratio for equal net work output and $P-V$ diagram of Stirling and Ericsson cycles for fixed temperature and volume limits and maximum pressure for equal net work output (inset); (b) non-dimensional net work as a function of volume ratio and temperature ratio (inset).

\section{Conclusions}

In this paper, Stirling and Ericsson cycles have been compared to determine which one produces more net work output for three different scenarios: (i) equal mass and temperature limits, where pressure and volume ratios are varied; (ii) equal mass and pressure or volume ratios, where the temperature limits are varied; and (iii) equal temperature and pressure or volume ratios, where the mass is varied.

For the first scenario, when both cycles are compared with equal working fluid mass and temperature limits, the Stirling cycle requires a higher pressure ratio than the Ericsson cycle to produce 
the equivalent net work output, and the Ericsson cycle requires a higher volume ratio than the Stirling cycle. Overall, this indicates that when the mass of the working fluid and the temperature limits are equal, an Ericsson engine produces more net work output if the pressure ratio is more limiting, and a Stirling engine produces more net work output if the engine size (volume) is a more critical design constraint.

For the first case of the second scenario, when both cycles are compared for a fixed low temperature value and with equal mass and pressure ratios, the Stirling cycle requires a higher high temperature value than the Ericsson to yield the same net work output. For the second case of the second scenario, when both cycles are compared for a fixed low temperature value and with equal mass and volume ratios, the Ericsson cycle requires a higher high temperature value than the Stirling to yield the same net work output.

For the first case of the third scenario, when both cycles are compared for a specific temperature ratio and maximum volume, the Stirling cycle produces more net work output above a threshold pressure ratio value, and when both cycles are compared for a specific pressure ratio and maximum volume, the Ericsson cycle produces more net work output above a threshold temperature ratio. In an example scenario, when the temperature ratio was fixed at 2.6, the Stirling cycle produces more net work output above a pressure ratio of 4.7 , and correspondingly, when the pressure ratio is fixed at 4.7 , the Ericsson cycle produces more net work output above a temperature ratio of 2.6. For the second case of the third scenario, when both cycles are compared for a specific temperature ratio and maximum pressure, the Ericsson cycle produces more net work output above a threshold volume ratio value, and when both cycles are compared for a specific volume ratio and maximum pressure, the Stirling cycle produces more net work output above a threshold temperature ratio. In an example scenario, when the temperature ratio was fixed at 2.6, the Ericsson cycle produces more net work output above a volume ratio of 4.7 , and correspondingly, when the volume ratio is fixed at 4.7 , the Stirling cycle produces more net work output above a temperature ratio of 2.6. The equations were derived to obtain these threshold values from the operating parameters.

The results in this paper reveal the various situations when one cycle produces more net work output than the other, which can be used to inform the design of the engines and determine when either a Stirling or Ericsson engine should be selected for a particular application.

Acknowledgments: The authors gratefully acknowledge the funding support from Transport Canada through a Clean Rail Academic Grant.

Author Contributions: B.D.M. conceived of the idea for the paper and supervised all of the work. R.F.C. performed the thermodynamic analysis and contributed ideas about how to organize and present the findings. R.F.C. and B.D.M. both wrote and edited the paper.

Conflicts of Interest: The authors declare no conflict of interest.

\section{Nomenclature}

$\begin{array}{ll}W & \text { Work }(\mathrm{kJ}) \\ P & \text { Pressure }(\mathrm{kPa}) \\ V & \text { Volume }\left(\mathrm{m}^{3}\right) \\ T & \text { Temperature }(\mathrm{K}) \\ m & \text { Mass of the working fluid }(\mathrm{kg}) \\ R & \text { Ideal gas constant }(\mathrm{kJ} / \mathrm{kg} \cdot \mathrm{K}) \\ \text { Subscripts } & \\ \text { max } & \text { Maximum } \\ \min & \text { Minimum } \\ H & \text { High } \\ L & \text { Low } \\ S & \text { Stirling } \\ E & \text { Ericsson }\end{array}$




\section{References}

1. Scollo, L.; Valdez, P.; Santamarina, S.; Chini, M.; Barón, J. Twin cylinder alpha stirling engine combined model and prototype redesign. Int. J. Hydrogen Energy 2013, 38, 1988-1996.

2. Campos, M.; Vargas, J.; Ordonez, J. Thermodynamic optimization of a Stirling engine. Energy 2012, 44, 902-910.

3. Cinar, C.; Yucesu, S.; Topgul, T.; Okur, M. Beta-type Stirling engine operating at atmospheric pressure. Appl. Energy 2005, 81, 351-357.

4. Sripakagorn, A.; Srikam, C. Design and performance of a moderate temperature difference Stirling engine. Renew. Energy 2011, 36, 1728-1733.

5. Cheng, C.H.; Yang, H.S.; Keong, L. Theoretical and experimental study of a 300-W beta-type Stirling engine. Energy 2013, 59, 590-599.

6. Paul, C.J.; Engeda, A. Modeling a complete Stirling engine. Energy 2015, 80, 85-97.

7. Çinar, C.; Karabulut, H. Manufacturing and testing of a gamma type Stirling engine. Renew. Energy 2005, 30, 57-66.

8. Parlak, N.; Wagner, A.; Elsner, M.; Soyhan, H.S. Thermodynamic analysis of a gamma type Stirling engine in non-ideal adiabatic conditions. Renew. Energy 2009, 34, 266-273.

9. Bert, J.; Chrenko, D.; Sophy, T.; Moyne, L.L.; Sirot, F. Simulation, experimental validation and kinematic optimization of a Stirling engine using air and helium. Energy 2014, 78, 701-712.

10. Kim, Y.; Chun, W.; Chen, K. Thermal-Flow Analysis of a Simple LTD (Low-Temperature-Differential) Heat Engine. Energies 2017, 10, 567.

11. Kongtragool, B.; Wongwises, S. A review of solar-powered Stirling engines and low temperature differential Stirling engines. Renew. Sustain. Energy Rev. 2003, 7, 131-154.

12. Thombare, D.; Verma, S. Technological development in the Stirling cycle engines. Renew. Sustain. Energy Rev. 2008, 12, 1-38.

13. Wang, K.; Sanders, S.R.; Dubey, S.; Choo, F.H.; Duan, F. Stirling cycle engines for recovering low and moderate temperature heat: A review. Renew. Sustain. Energy Rev. 2016, 62, 89-108.

14. Sisman, A.; Saygin, H. On the power cycles working with ideal quantum gases: I. The Ericsson cycle. J. Phys. D Appl. Phys. 1999, 32, 664.

15. Bonnet, S.; Alaphilippe, M.; Stouffs, P. Energy, exergy and cost analysis of a micro-cogeneration system based on an Ericsson engine. Int. J. Therm. Sci. 2005, 44, 1161-1168.

16. Touré, A.; Stouffs, P. Modeling of the Ericsson engine. Energy 2014, 76, 445-452.

17. Creyx, M.; Delacourt, E.; Morin, C.; Desmet, B. Dynamic modelling of the expansion cylinder of an open Joule cycle Ericsson engine: A bond graph approach. Energy 2016, 102, 31-43.

18. Kaushik, S.; Kumar, S. Finite time thermodynamic evaluation of irreversible Ericsson and Stirling heat engines. Energy Convers. Manag. 2001, 42, 295-312.

19. Tyagi, S.K.; Kaushik, S.C.; Salhotra, R. Ecological optimization and performance study of irreversible Stirling and Ericsson heat engines. J. Phys. D Appl. Phys. 2002, 35, 2668.

20. Bădescu, V. Optimum operation of a solar converter in combination with a Stirling or Ericsson heat engine. Energy 1992, 17, 601-607.

21. Creyx, M.; Delacourt, E.; Morin, C.; Desmet, B.; Peultier, P. Energetic optimization of the performances of a hot air engine for micro-CHP systems working with a Joule or an Ericsson cycle. Energy 2013, 49, 229-239.

22. Wojewoda, J.; Kazimierski, Z. Numerical model and investigations of the externally heated valve Joule engine. Energy 2010, 35, 2099-2108.

23. Feidt, M.; Costea, M. Energy and Exergy Analysis and Optimization of Combined Heat and Power Systems. Comparison of Various Systems. Energies 2012, 5, 3701-3722.

24. Hachem, H.; Creyx, M.; Gheith, R.; Delacourt, E.; Morin, C.; Aloui, F.; Nasrallah, S.B. Comparison Based on Exergetic Analyses of Two Hot Air Engines: A Gamma Type Stirling Engine and an Open Joule Cycle Ericsson Engine. Entropy 2015, 17, 7331-7348.

25. Eames, I.W.; Evans, K.; Pickering, S. A Comparative Study of Open and Closed Heat-Engines for Small-Scale CHP Applications. Energies 2016, 9, 130.

(C) 2018 by the authors. Licensee MDPI, Basel, Switzerland. This article is an open access article distributed under the terms and conditions of the Creative Commons Attribution (CC BY) license (http:/ / creativecommons.org/licenses/by/4.0/). 